\title{
An Introduction to Vascular Biology
}

\section{By A. Hadley et al. \\ Cambridge University Press, \$85, 286 pp. ISBN 0-521-58998-3, 1998}

ReViewed by Russell Ross

Department of Pathology

Director, Center for Vascular Biology,

School of M edicine, University of W ashington Seattle, W A 98195, USA

An Introduction to Vascular Biology is a multi-authored text, which, according to the introduction, is divided into three areas: development and physiology; atherosclerosis, hypertension, and diabetes; and other specific pathological problems. The individual chapters are wellwritten and range in content from introductory material to fairly detailed information, such as that dealing with atherosclerosis, restenosis and the contractile mechanisms of smooth muscle.

However, the organization of the book is somewhat peculiar. For example, the first part, which deals with development and control of blood vessel function, provides little information on embryogenesis and vasculogenesis of the cardiovascular system in general. The first chapter on angiogenesis is well-written but, because there is no discussion of vasculogenesis, the book ignores the interesting literature on smooth muscle cells and their role in the development of the vascular system. Smooth muscle cells that envelop the arteries and arterioles in different parts of the vascular tree are derived from different embryogenic sources; this may help to explain differences in smooth muscle responsivity in diseases as they occur at different levels in the arterial system.

The remaining chapters in the first section cover flow-mediated responses and endothelial control of vascular tone and permeability, as well as contraction of smooth muscle and neurohumoral regulation of tone. They are relatively up-todate, serve as good introductions to these areas of interest and lead the reader to recent references.

The second part of the book concentrates on hypertension, atherosclerosis and diabetes. These chapters are remarkably up-to-date for an introductory text and provide excellent references and a conceptual framework that helps to relate

structure and functional changes (as, for example, in hypertension), with mechanisms of atherosclerosis and with problems that result from atherosclerosis, which include restenosis and aneurysm formation, as well as atherosclerosis as a complication of diabetes.

The book's third section deals with additional clinical pathologic problems and includes reasonably comprehensive and interesting chapters on wound healing, alterations in endothelial function in inflammation and sepsis, and the role of the endothelial cells in transplant rejection.

The book was written to introduce vascular biology to individuals investigating physiology. These may be physicians, Ph.D. students, and, as the authors state, "those who are curious about how blood vessels and endothelium develop, perform, and misbehave." It certainly achieves the goal of introducing this fascinating and important area of endeavor to these and others, and is referenced sufficiently well to lead readers to specialized areas of interest.

The editors might have taken the more

\section{Viruses, Plagues, and History}

\author{
by Michael B. A. Oldstone \\ Oxford University Press, \$25, 288 pp
}

ISBN: 0-19511-723-9, 1998

Reviewed by Geoffrey L. Simith

Sir W illiam Dunn School of Pathology University of Oxford

South Parks Road

Oxford OX1 3RE, UK

I enjoyed reading this book. It provides a vivid description of several terrible viral diseases that have in some cases been controlled by vaccination to the point of extinction (e.g. smallpox), while in others rage unchecked causing misery to countless millions (e.g AIDS). The book brings together not just a description of these diseases and how they have affected our history and behavior, but also narrates the search for the causative agents, explaining how they are transmitted, how research led to the develop- mundane but perhaps more useful approach of dividing the book into three areas, for example: 1, development, which would cover vasculogenesis and angiogenesis of the vascular system (missing from the book, leaving the reader with questions); 2 , cells of the vascular system, including endothelium, smooth muscle, myocardium, other connective tissue cells, and blood cells that interact with the vascular tree, including leukocytes and platelets; and 3, the diseases of the cardiovascular system that have been covered in the text. This then could have provided opportunities for discussions of cellular interactions and set the stage for discussions of diseases. Nevertheless, most of the chapters are well-written, and the illustrations are helpful and add to the value of the book.

Thus, despite the shortcomings noted above, An Introduction to Vascular Biology serves as a good introduction to the subject and can be a worthwhile resource for those interested in vascular biology and looking to pursue its interesting components.

ment of vaccines, and how these vaccines have controlled specific diseases. Most importantly, the book introduces the scientists involved in these endeavors, so that the reader is left with an understanding of the characters and lives of those whose research has been of great benefit to mankind, and who in some cases paid the ultimate price in their work.

The author has made a clear effort to make the subject understandable to those outside biomedical research and he has achieved this goal. Thus the book will be of general interest to many without detailed knowledge of viruses, the immune system or vaccines. Yet the book is also an excellent read for health care workers, biomedical students, scientists, virologists, immunologists and those interested in the history of man's battle with infectious disease.

After a general introduction there are short chapters about the principles of virology and immunology, including explanations of how viruses replicate, how our immune system controls and eliminates viral infections and how some 